\title{
W.
}

\section{Development of an automated wheelchair guided by a magnetic ferrite marker lane}

\author{
Hiroo Wakaumi, BEng; Koichi Nakamura, BEng; Takayoshi Matsumura, BEng \\ Resources and Environment Protection Research Laboratories, NEC Corporation, Kawasaki 213 Japan; \\ EDP Transportation Industry Systems Division, NEC Corporation, Tokyo 108-01 Japan; System Development \\ Division, NEE Ltd., Tokyo 108 Japan
}

\begin{abstract}
An automated wheelchair with a guide sensor, guided by a magnetic ferrite marker that is resistant to the presence of dirt, is described. The wheelchair permits the severely disabled, as well as the older population, to move about freely, both indoors and outdoors. This is due to the simple operation involved (pushing a button), and a magnetic ferrite marker lane which is minimally influenced by dirt or other nonmagnetic materials. For increased comfort, a nonlinear signal-processing circuit and pulse-steering drive method have been developed to provide a smooth running operation. In addition, a function that provides for stopping at desired destinations has been added for convenience, and a collision prevention function using infrared sensors has been added for safety.
\end{abstract}

Key words: automated wheelchair, biomechanics, ferrite marker, magnetic sensor.

\section{INTRODUCTION}

In response to the demands of wheelchair users for equal access, hand-propelled wheelchairs, electricallycontrolled wheelchairs, and automated-guided wheelchairs (AGW) have been developed. However, because upper body strength is required, a hand-propelled wheelchair does not permit an older or severely disabled person an extensive range of travel. An electrically-controlled wheelchair can be controlled by a manually-operated joystick - but because this type of wheelchair sometimes zigzags, and a slight

Address all correspondence and requests for reprints to: Mr. Hiroo Wakaumi, Resources and Environment Protection Research Laboratories, NEC Corporation, 4-1-1, Miyazaki, Miyamae-ku, Kawasaki 213, Japan. movement of a joystick can cause a quick turn, driving an electrically-controlled wheelchair requires the operator to be skilled both in turning, and in direction-change operations-especially on narrow or curved roads. Therefore, it is difficult for many severely disabled and/or elderly persons to operate them skillfully.

Conventional AGWs, which are guided by reflective tape markers laid on the road or floor, are influenced by dirt on the tape. That is, when reflective tape markers are coated with dirt or mud, the photo-detection sensor (with photo diodes) installed on the wheelchair cannot discriminate these markers from the surrounding road or floor surface; therefore, the user cannot control the steering wheel, making this type of wheelchair difficult to use.

In addition to the photo-detection guidance technique for the AGWs, other automatic vehicle guidance techniques, such as visual machine guidance and buried-wire guidance systems might be used (1). As a visual machine guidance technique, optical guiding along a painted track, using a video camera, has been proposed (2). Since much processing time is necessary for this guidance system to recognize the position of the track in front of the AGW, it is impossible to move very fast; this technique is also not useful in a dirty, rain-covered, or snowy environment, because the camera cannot recognize the track covered with dirt or snow.

When using a buried-wire guidance system, if the wire is cut for some reason, it cannot operate due to lack of a magnetic field generation. Changes in the guidance lane location are awkward because the wire must be buried. Therefore, in addition to a high implementation cost, this guidance system lacks reliability. 


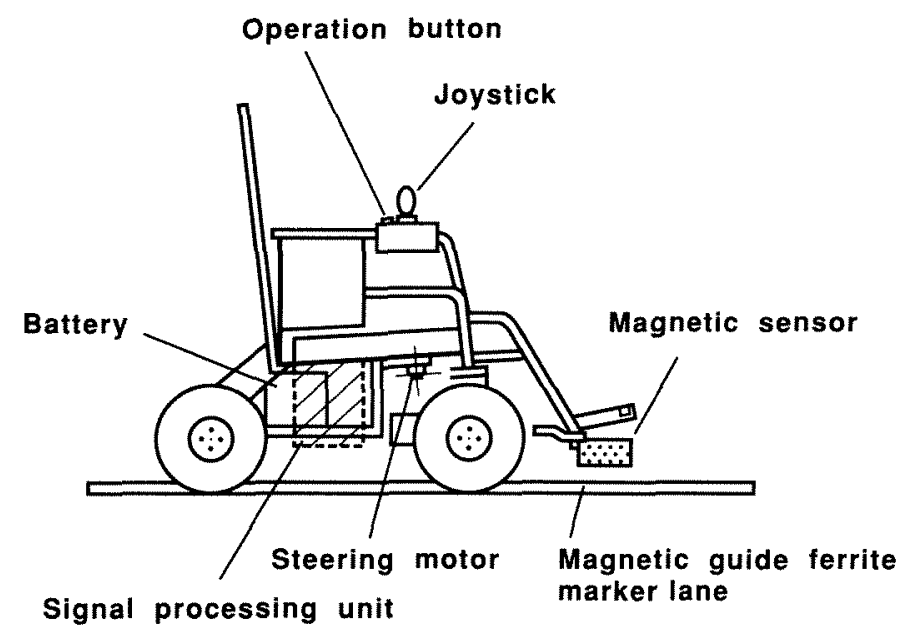

Figure 1.

Automated guided wheelchair configuration.

The magnetic ferrite marker technique is useful because the vehicle can move relatively quickly (due to simple recognition of the marker position by magnetic sensors) and is not influenced by a local marker being cut off (3).

This paper describes an automated wheelchair guided by a magnetic ferrite marker which is relatively free from adverse influence by dirt or mud and because of its simple operation, permits easy use by severely disabled and older people (4). This wheelchair would be especially useful for mentally alert people with severe motor impairment (e.g., quadriplegics and cerebral palsy patients), since it allows them to move wherever they want to go along a laid- out route, merely by a simple push-button operation. A nonlinear circuit and a pulse-steering drive method (developed to achieve a smooth running operation), an infrared sensor system for stop operation, and safety functions are described. Investigation results were derived from the running characteristics of this wheelchair.

\section{GUIDANCE TECHNIQUE PRINCIPLE}

Figure 1 shows an AGW with a magnetic sensor guided by magnetic ferrite markers which are laid in/on the sidewalk or floor $(3,5)$. Any electric motor-driven wheelchair can be partially modified by installing the magnetic sensor, signal-processing circuit, and operation button, allowing a change in its mode of operation. The magnetic sensor is installed under the footrests at the front of the wheelchair to control the steering wheels. This sensor, which is $7 \mathrm{~cm}$ from the road surface or floor, picks up guidance signals from magnetic ferrite markers. The magnetic ferrite marker lane, using soft ferrite material bound in place with resin, is $10 \mathrm{~cm}$ wide, $5 \mathrm{~mm}$ thick, and can be extended as far as necessary.

Figure 2a shows the magnetic sensor and ferrite marker configuration. Figure $\mathbf{2} \mathbf{b}$ shows the magnetic sensor signal-processing circuit. The magnetic sensor consists of an exciting coil, $L$, at the center of the sensor unit and two detecting coils, $L l$ and $L 2$, placed on its right and left sides. Exciting coil $L$ generates a magnetic field. The ferrite marker is magnetized by this field and sets up a new resonant magnetic field; the result being that the original magnetic field is deviated. Detecting coils, $L I$ and $L 2$, pick

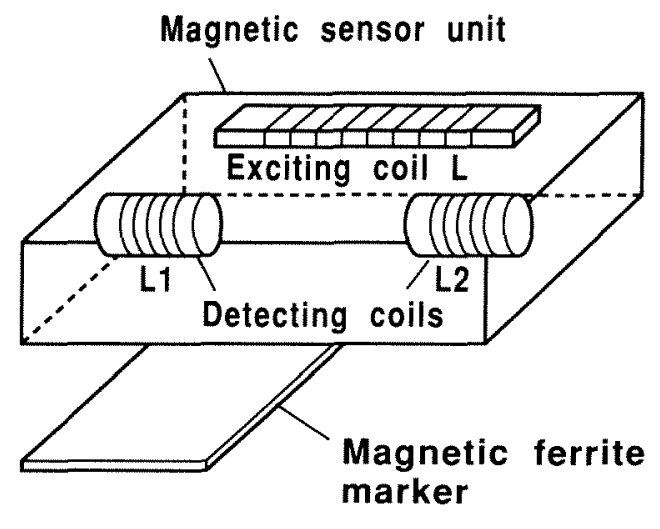

(a)

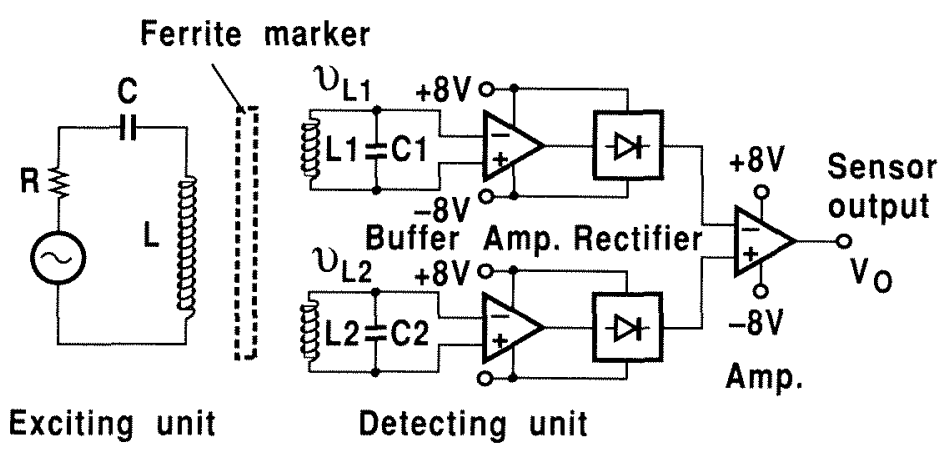

(b)
Figure 2a.

Magnetic sensor: System configuration.
Figure 2b.

Magnetic sensor: Signal-processing circuit. 


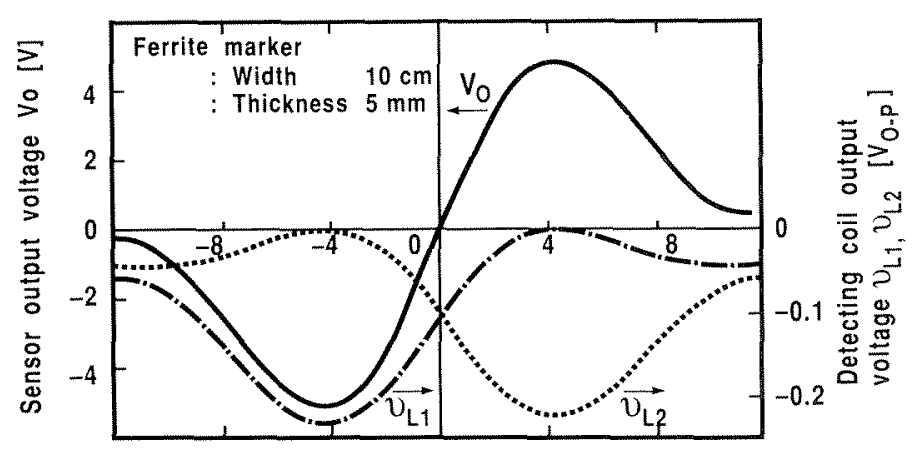

Sensor position from center of magnetic ferrite marker [cm]

Figure 3.

Detecting coil- and sensor-output voltage versus sensor position.

up the magnetic field deviation. The detected output signals obtained by two detecting coils, $\mathrm{V}_{\mathrm{L} 1}$ and $\mathrm{V}_{\mathrm{L} 2}$, reduce linearly, as shown in Figure 3, as the sensor position deviates from the center of the marker lane. These signals reach a peak level and then reduce to a small value as the sensor position deviates further. These characteristic signals cross at the center of the marker lane. These output signals are subtracted from each other to obtain an S-shaped characteristic, suitable for use as a steering control signal. As a result, since the sensor output signal (i.e., the difference between these detection-output signals), is proportional to the wheelchair deviation from the center of the ferrite marker lane in a line or curved form, the difference signal permits controlling the movement direction of the wheelchair. For example, consider the case where the sensor position deviates slightly from the center of the marker. When the sensor output signal voltage is increasing to a higher positive level, a controller for governing the steering motor rotation direction permits it to rotate the forward wheels in such a direction as to bring the sensor position back to the center of the marker lane. When the sensor output voltage is decreasing to a lower negative level, the controller permits the forward wheels to rotate in the opposite direction. Thus, the wheelchair can be controlled in a route approximately along the center of the marker lane by using the magnetic sensor.

\section{NEW TECHNIQUES FOR HIGH LEVEL PERFORMANCE}

Safety, comfort, and convenience are very important for wheelchair users: therefore, several considerations are required to realize a high-level performance wheelchair system.

\section{Smooth running operation for comfort}

In order to provide a comfortable ride, a partially steering-free operation by a nonlinear circuit and pulsesteering drive method was developed.

In general, when the wheelchair's steering is controlled according to a sensor output signal indicating sensor positions (steering angle changes linearly with the sensor output signal), the wheelchair movement tends to oscillate slightly. This oscillation phenomenon occurs when a system, including the sensor, signal-processing circuit, controller, drive motor, drive wheels, and magnetic marker, is set up for a certain oscillation condition. The magnitude of the steering angle changes almost linearly with the sensor output signal. Since a steering control signal (sensor output signal) is applied continuously to the wheelchair, it is almost immediately brought back toward the center of the route whenever it deviates slightly from the center of the marker. In this case, the wheelchair could move from side to side at a certain variation rate (a relatively higher perturbation frequency than a few cps). When this return operation frequency matches and reinforces a system oscillation frequency, the result is the system oscillation frequency being different from the ordinary intermittent and irregular steering control state. Once the system starts spurious oscillating, it is difficult for the system to shed this movement variation.

To prevent this wheelchair from wobbling during operation, a steering-free operation within a small deviation range (i.e., a small area along the center of the ferrite marker lane when the magnetic sensor deviates slightly) is realized by a nonlinear signal-processing circuit (Figure 4). This circuit consists of an amplifier and several diodes, used to shift about 1 to $2 \mathrm{~V}$ of the sensor output voltage point, when the steering control voltage rises. When switch $\mathrm{A}$ is on, about $\pm 1 \mathrm{~V}$ steering-free operation voltage range is obtained. When both switches $\mathrm{A}$ and $\mathrm{B}$ are off, about $\pm 1.5 \mathrm{~V}$ steering-free operation voltage range is obtained. Even when the magnetic sensor deviates within these steering-free operation ranges, accurate steering control is not immediately achieved. In this case, system steering control oscillation is retarded, because the return

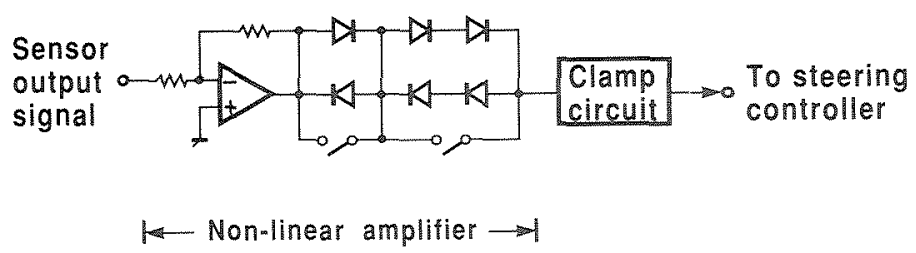

Figure 4.

Nonlinear signal-processing circuit configuration. 


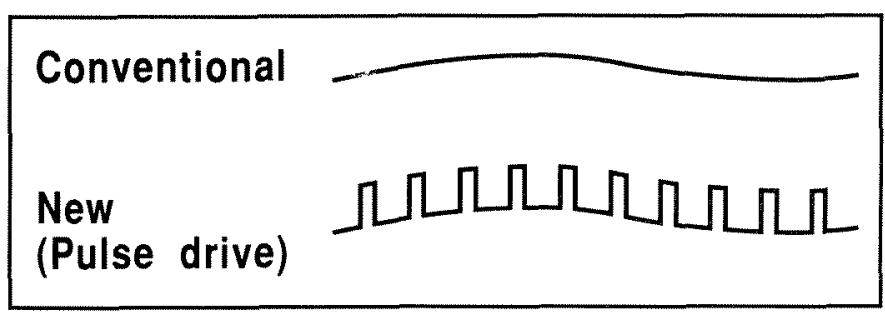

Figure 5.

Steering drive pulse in pulse-steering drive method, compared with conventional drive method.

operation from side to side becomes slower than the oscillation, enhancing safe driving.

The wheelchair also has a tendency to zigzag (not oscillate) due to any quick movements of the steering wheel when partially steering-free operation is achieved by the nonlinear circuit. That is, after the steering angle rises sharply when steering control starts in a conventional continuous steering drive method, subsequent steering movements continue to be controlled within the steering operation ranges. Therefore, the steering wheel movement sometimes overruns to the other side and follows a zigzag course. If the wheel movement inertia moment is too large at a subsequent change in its movement direction by the steering control signal, the wheelchair cannot change this moment direction immediately. This zigzag running is a slow movement with a large arc locus and differs from the above mentioned oscillating operation.

To minimize this zigzag running to the smallest extent, a new pulse-steering drive method is used within a small steering wheel deviation range. With the pulse-steering drive method (Figure 5), intermittent drive pulses are added to the sensor output signal and passed through the nonlinear circuit. As a result, steering is gradually changed from a lower sensor output voltage than that required for the steering-free limit (does not cause steering to change greatly when steering control starts). The intermittent drive pulse variation consists of a positive pole pulse, when the sensor output signal is positive, and a negative pole pulse, when the sensor output signal is negative. Its variation frequency is 10 times per second (with a 10 percent to 25 percent duty range). With this drive method, a gradual steering control is achieved. When the wheelchair deviates a little from the center of the marker lane, the control steering angle is set relatively small. As it deviates further from the marker lane center, the steering control angle becomes relatively greater. Thus, a delicate steering wheel angle control is possible and is not expected to cause overrun. As a result, the wheelchair will move smoothly without zigzag running.

\section{Automatic stop operation for convenience}

People who use wheelchairs may be severely visuallydisabled or lack the full use of their hands. When the user needs to move to several destinations in sequence, the automatic stop operation is required at specified positions. (This route can be set up before wheelchair movement starts.) To permit the wheelchair to stop automatically at desired destinations, an infrared position detection sensor system has been constructed which detects the reflection tape previously placed at the destination (laid on the floor or sidewalk). The reflecting tape material has a different reflection coefficient from that of the surrounding floor or pavement surface. In experiments, road sign reflecting material now on the market was used as reflecting tape. When the position detection sensor on the wheelchair detects this reflecting tape, it automatically stops the wheelchair movement.

When there are several destinations, the sensor counts the number of tape markers as the wheelchair passes them so that the user can move easily to a desired destination.

\section{Emergency stop operation for safety}

Maintaining safety is important for every user. To prevent a possible collision with people, chairs, animals, etc., two infrared obstacle detection sensors were constructed on the front of the wheelchair. When an obstacle appears in front of the wheelchair, it detects the obstacle, stops, and remains stationary until the obstacle is moved out of the path of the wheelchair.

Figure 6 shows a control circuit block diagram. A selection switch used to select either automatic mode or manual mode is provided for achieving flexible movement. (For example, the manual mode allows users to operate the wheelchair even in locations where the marker lane has not been laid out.) In the automatic mode, an automaticmanual selection switch circuit permits current to flow in an electromagnetic relay and selects the automatic mode. In this case, the magnetic sensor output signal is used for steering control through the nonlinear circuit and the pulse generator. Also, the velocity, predetermined in a velocity regulation part, is used for drive motor speed control (front wheel movement speed control).

However, when an obstacle or position detection sensor detects either an obstacle in the path or reflecting tape laid at the destination, the operation mode is automatically changed to manual mode and the wheelchair stops. The joystick is held in the center of its control area and steering-and drive-motor control signals, supplied from a handle operation, are not present. In the manual mode, steering and speed are completely controlled by operating the joystick. 


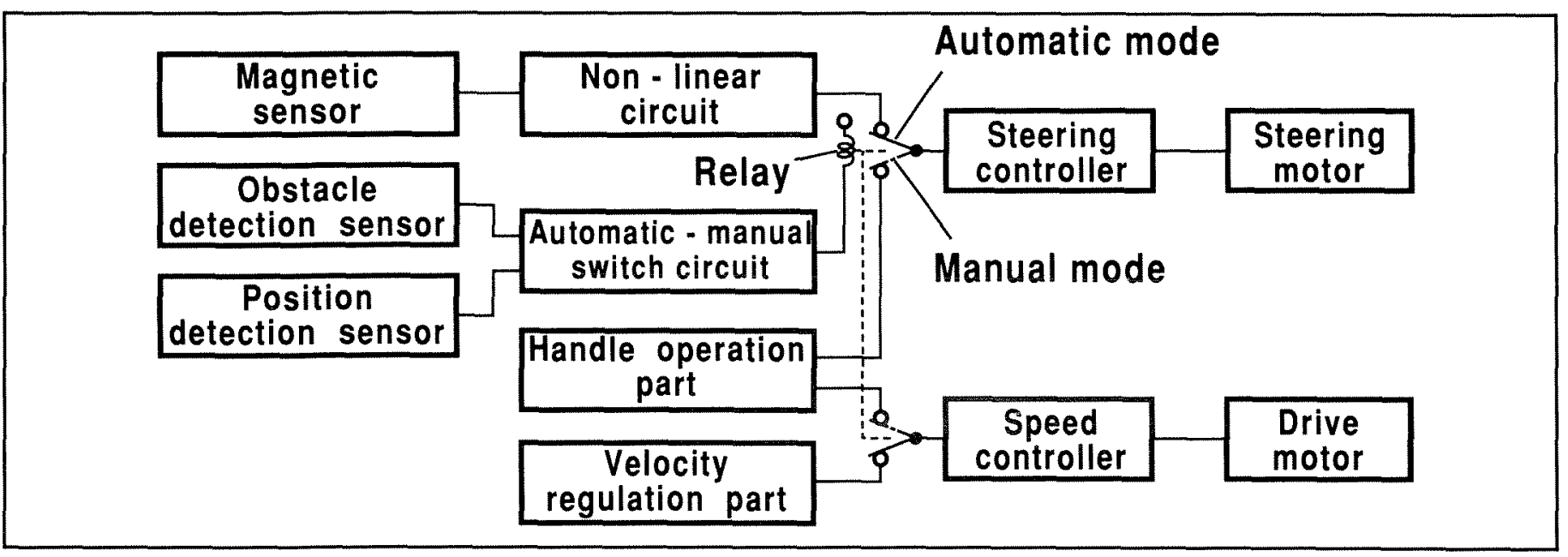

Figure 6.

Control circuit block diagram.

\section{RESULTS}

The automated wheelchair, with the magnetic sensor on the front (under the footrests) is shown in Figure 7. Running experiments were carried out along a corridor with a soft ferrite marker on the floor. Figure 8 shows the infrared position-detection sensor installed on the left side of the wheelchair (about $25 \mathrm{~cm}$ above the floor), and the reflection tape marker laid on the floor. When the automated mode is selected, the wheelchair movement is started by pushing a button which has been installed on the back of the joystick console box.

Figure 9 shows the steering angle versus the sensor output voltage, realized by using the nonlinear circuit and the pulse-steering drive method. Circuits 1 and 2 have different gains and their diodes have nonacting zones in the nonlinear circuit, giving $\pm 0.9 \mathrm{~V}$ and $\pm 1.4 \mathrm{~V}$ sensor output steering-free operation in the voltage area. The wheelchair did not oscillate in either case, since steering was not controlled within these areas. However, a wheelchair with such a nonlinear circuit showed zigzag running when gradual steering controls were not used.

The gradual steering control method caused a steering angle change, gradually changing from about $\pm 0.5 \mathrm{~V}$. The wheelchair with this gradual steering control method did not cause overrun (zigzag running). In this case, it successfully ran along the marker at less than $4 \mathrm{~km} / \mathrm{h}$, even when carrying a $180 \mathrm{~kg}$ load. At higher speeds, a slight zigzag running phenomenon was observed, which should improve in the future.

When an obstacle appeared about 2 meters in front of the wheelchair (the infrared sensor-detection range), it

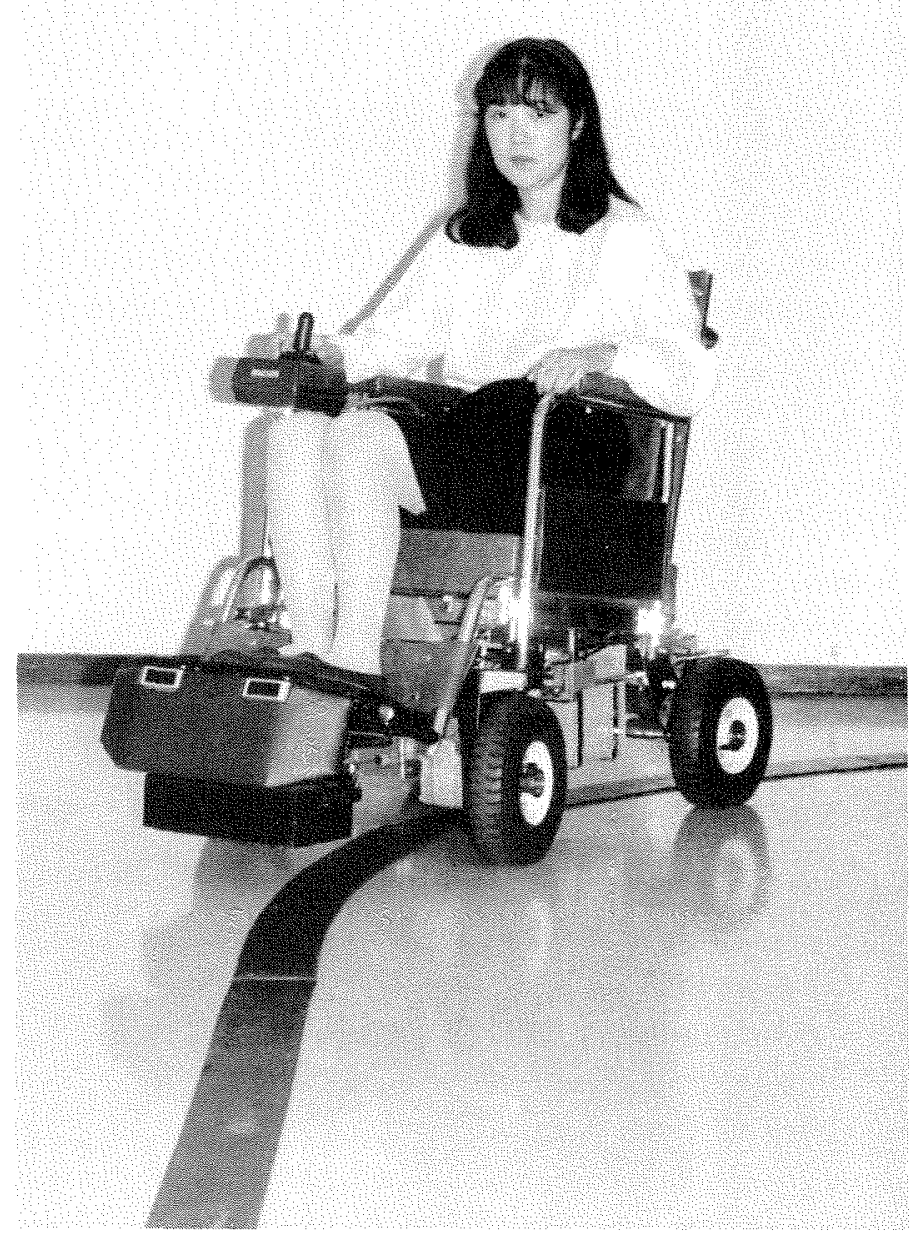

Figure 7.

Automated wheelchair during running experiment. Two infrared obstacle detection sensors are installed at the front. 


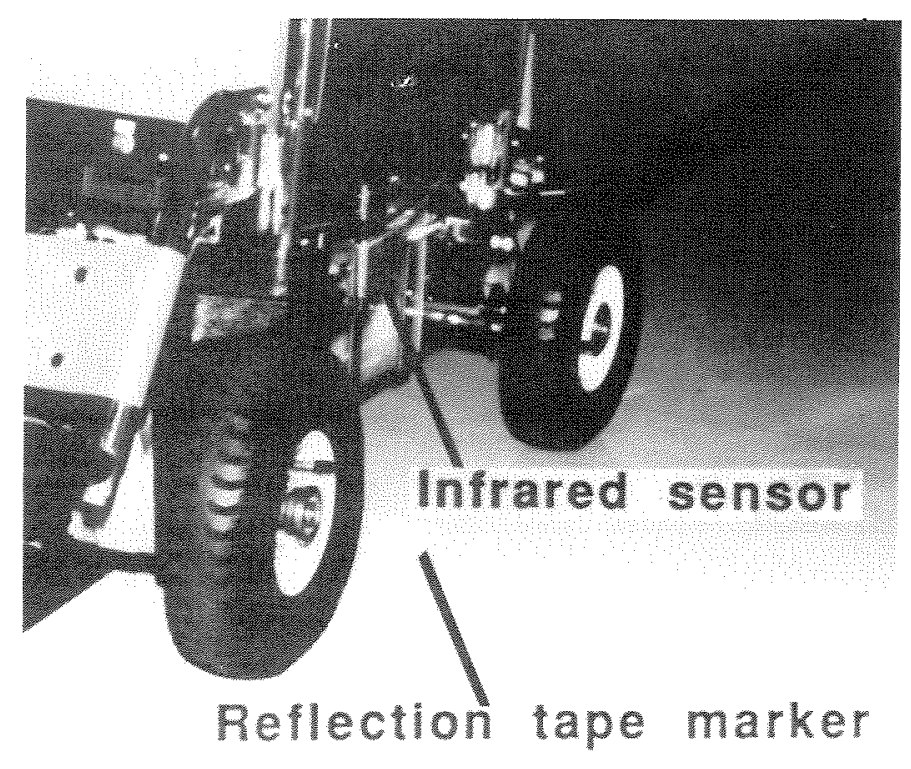

Figure 8.

Infrared position detection sensor and reflecting tape marker used to stop the wheelchair at specified positions.

came to a stop safely. The wheelchair also comes to a stop at a predetermined position along a set route when detecting previously-laid reflection tape.

Thus, this wheelchair moved without zigzag at less than $4 \mathrm{~km} / \mathrm{h}$ walking speed, and automatically stopped at the desired destination; also, obstacles were accurately detected and collision safely prevented. This wheelchair system was exhibited at the Second International Home Car \& Rehabilitation Exhibition held in Japan in 1988, and many people experienced its performance at less than $4 \mathrm{~km} / \mathrm{h}$ along a track route with a $2.5 \mathrm{~m}$ radius. Specifications including other features for the automated wheelchair are shown in Table 1.

\section{DISCUSSION}

Because the magnetic ferrite marker is used as a guide lane and has a strong magnetic property, it becomes momentarily magnetized by an alternate magnetic field (about $40 \mathrm{kHz}$ ). The resonant field can be picked up by the magnetic sensor without being influenced by dirt or small nonmagnetic materials left around and over the ferrite marker. This characteristic is different from that of the photo-detection technique (with photo diodes) used for a conventional AGW. A Charge Coupled Device (CCD) camera system can be used as another sensor in the photodetection technique. However, because this system picks up the same signal as the conventional reflective tape marker, an AGW with this CCD camera system is easily influenced by many kinds of dirt coated or dotted on the marker.

Dynamic modeling of the wheelchair system using microprocessors was investigated $(6,7)$. In this wheelchair system, it is necessary to construct a software program for computing control algorithms and sending steering control signals to a steering motor control part. The initial development cost for the software program seems to be high. On the contrary, an analog controller using the nonlinear circuit is easily constructed and its development cost is low. However, if the packaging of software for the steering control system with microprocessors progresses, the development cost will be reduced in the future and may be applied to this automated wheelchair with a magnetic sensor for outdoor use.

This wheelchair is available for forward or turn-back movement along a single guidance route (a ferrite marker lane). Performance is sufficient for practical use, especially outdoors. When microcomputers are installed in the future, the wheelchair will be able to move along a more flexible route. For example, even if there were some branch points along one marker lane, the wheelchair can be controlled to move anywhere the user would like to go by detecting markers at the branch position.

Also, even when the routes are composed of alternate ferrite marker lanes, the wheelchair can run along such routes. In this case, in the gap between two adjacent marker lanes, the wheelchair moves autonomously with the steering control (without the detection of the magnetic marker).

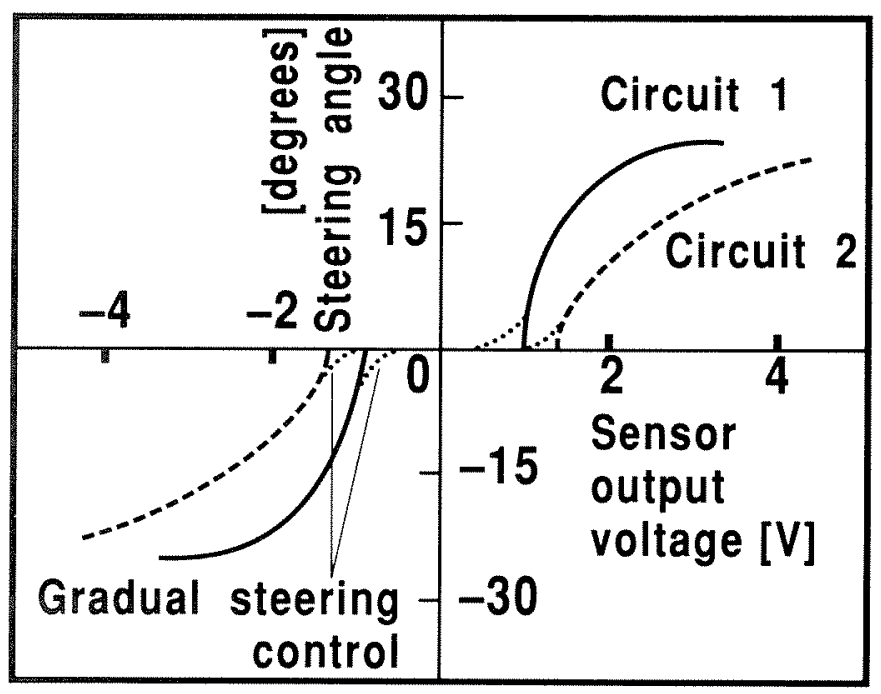

Figure 9.

Steering angle versus sensor output voltage characteristics determined by a nonlinear circuit and a pulse-steering drive method (gradual steering control). 
Table 1.

Newly developed wheelchair specifications.

\begin{tabular}{|c|c|c|}
\hline No. & Item & Specifications \\
\hline 1 & Guidance & Magnetic guide method with ferrite marker lane \\
\hline 2 & Movement mode & Automatic mode (manual mode possible) \\
\hline 3 & Drive method & Front wheel direct drive \\
\hline 4 & Drive control method & 3-stage speed change by switch \\
\hline 5 & Brake & Electric brake and dynamoelectric damping brake by drive motor \\
\hline 6 & Steering control & Front wheel power steering \\
\hline 7 & Practical uphill angle & About 7 degrees \\
\hline 8 & Maximum level difference to be overcome & About $6 \mathrm{~cm}$ \\
\hline 9 & Minimum rotation radius & 2.5 meters \\
\hline 10 & Running speed & Max. $2.2 \mathrm{~km} /$ hour \\
\hline 11 & Battery & $12 \mathrm{~V} \times 2$ \\
\hline 12 & Battery charge & Automatic charging method with electronic timer \\
\hline 13 & Drive motor & Max. output power $24 \mathrm{~V} 180 \mathrm{~W} \times 2$ \\
\hline 14 & Weight & About $70 \mathrm{~kg}$ \\
\hline 15 & External dimensions & $122.5 \mathrm{~cm}$ Length $\times 56 \mathrm{~cm}$ Width $\times 78 \mathrm{~cm}$ Height \\
\hline 16 & Permission running time on flat areas & About 4 hours \\
\hline
\end{tabular}

Since this system depends on detecting a route for movement control, when it cannot detect a sensor output signal within a certain distance, this gap is limited to a shorter distance than the minimum moving distance in the course. When the wheelchair changes the present route to some other route, it detects crosspoints on the ferrite marker lanes at possible route change locations. It then moves along the route in the desired direction. By using these alternate routes, the system cost reduces to a point lower than the cost for a single route. In these cases, the activity range for users will be greatly increased.

\section{CONCLUSION}

An automated wheelchair was developed with a magnetic sensor used for guidance. It was automatically guided by a magnetic ferrite marker laid in/on the sidewalk or floor. This AGW allowed the user to move about by merely pushing a button. Due to the partially steering-free operation, realized by a nonlinear circuit and a pulse-steering drive method, it showed a satisfactory high-running performance (safe, comfortable, and stable-running). Con- venient automatic stop functions used at desired destinations were the result of an infrared sensor for detecting a reflecting tape marker, as well as for stopping at prescribed positions. A temporary stop function for assuring driving safety resulted from providing two infrared sensors for obstacle detection and for stopping.

Because the ferrite marker is not influenced by dirt or other small nonmagnetic materials on the marker, it is applicable both indoors and out of doors. This easily operated wheelchair using such a marker increases the activity range for users. This will be especially important in the future, when the average age of the user will be higher.

\section{ACKNOWLEDGMENTS}

The authors would like to thank I. Sugano, K. Matsumi, and $F$. Yamauchi for their support and encouragement. They also would like to thank Dr. H. Makino for his useful discussion. Thanks also are due to Mr. Y. Sato, who produced the signalprocessing circuit. 


\section{REFERENCES}

1. Fenton RE, et al. One approach to highway automation. In: Proceedings of IEEE, New York, March, 1968. Vol. 56. New York: IEEE, 1968:556.

2. Jullieri $\mathrm{M}$, Bazin B. Video guiding of an industrial cart. In: Proceedings of the 6 th International Conference, AGVS-6, Brussels, Belgium, October, 1988. 1988:171-8.

3. Kondo Y, Miura T, Ito A, Fujimoto J, Yamauchi F. Magnetic marker systems using by-product ferrite. In: ICF-4, 1984;589-593.

4. Wakaumi H, Nakamura K, Matsumura T, Yamauchi F. Automated wheelchair guided by magnetic ferrite marker. In: Presperin JJ, ed. Proceedings of the 12th Annual RESNA Conference, New
Orleans, LA, June 25-30, 1989. Washington, DC: RESNA PRESS, 1989:47-48.

5. Sakairi N, Yamada H. Ferrite sensor for automated ferrite guided vehicle. NEC Tech J 1988;41:78-80 (in Japanese).

6. Johnson BW, Aylor JH. Dynamic modeling of an electric wheelchair. IEEE Trans Ind Appl 1985;IA-21:1284-1293.

7. Brown KE, Inigo RM, Johnson BW. Design, implementation, and testing of an adaptable optimal controller for an electric wheelchair. In: Proceedings of the IEEE Industry Applications Society Annual Meeting, San Diego, October, 1989. New York: IEEE, 1989:2332-9. 